\title{
TRANSEXUALIDAD Y ADOLESCENCIA: UNA REVISIÓN SISTEMÁTICA
}

\author{
Olga Fernández-García \\ Dpto de Psicología Evolutiva y de la Educación, Universitat de València, Valencia, España \\ Rafael Ballester-Arnal \\ Departamento de psicología Básica, Clínica y Psicobiología, Universitat Jaume I de Castellón, España \\ Paula Iglesias Campos \\ Dpto de Psicología Evolutiva y de la Educación, Universitat de València, Valencia, España \\ Vicente Morell-Mengual \\ Dpto de Psicología Evolutiva y de la Educación, Universitat de València, Valencia, España \\ María Dolores Gil-Llario \\ Dpto de Psicología Evolutiva y de la Educación, Universitat de València, Valencia, España \\ feranol@alumni.uv.es
}

Fecha de Recepción: 28 Marzo 2018

Fecha de Admisión: 10 Abril 2018

\section{RESUMEN}

El estudio de la transexualidad ha evolucionado en los últimos años alcanzando mayor visibilidad actualmente gracias a las iniciativas de los colectivos trans y al incremento de niños, niñas y adolescentes que acuden a atención primaria por problemas de identidad de género. Este creciente interés ha estimulado la investigación cuya abundancia precisa un análisis y selección de los trabajos más relevantes. Con este objetivo hemos realizado una revisión bibliográfica con el fin de abordar el estado actual de la transexualidad temprana, centrándonos en los estudios relativos a su etiología, prevalencia, diagnóstico y tratamientos actuales. Para ello se consultaron diversas bases de datos (Web of Science, PubMed, PubPsych, Elsevier, PsycINFO, PsycARTICLES, Psychology Database) restringiendo la búsqueda por palabras clave ("transsexuality", "transsexualism", "adolescence", "adolescents" y "teenagers") y fecha de publicación (2008-2017). Inicialmente se obtuvieron 103 de los cuales fueron descartados 66 trabajos por tratar aspectos que no eran objeto de la revisión y exponer resultados no generalizables por su reducida muestra. Del análisis de los 37 artículos seleccionados se obtiene, por lo que se refiere a la etiología, que la transexualidad tiene un origen heterogéneo y multifactorial. La prevalencia obtenida es del 9.8\%. En el diagnóstico se insiste en la necesidad de realizar un seguimiento permanente haciendo uso de la entrevista como principal instrumento de elección. Por último, la mayoría de investigaciones resaltan la importancia del nuevo abordaje terapéutico basado en la terapia triádica que contempla tratamiento psicológico (asimilación del rol deseado de forma plena), hormonal (bloqueo hormonal y tratamiento con hormo- 


\section{TRANSEXUALIDAD Y ADOLESCENCIA: UNA REVISIÓN SISTEMÁTICA}

nas cruzadas) y quirúrgico (cambio de genitales y otros caracteres sexuales), a pesar de la controversia que ha suscitado por los posibles efectos desfavorables para la población infanto-juvenil. Se concluye la necesidad de continuar investigando para lograr no solo un diagnóstico y tratamiento más individualizado sino una mayor divulgación de conocimiento que ayude a disminuir la estigmatización.

Palabras clave: transexualidad; adolescencia; proceso transexualizador

\section{ABSTRACT}

Transexuality and adolescence: a systematic review.

The study of transsexuality has evolved in recent years reaching greater visibility now thanks to the initiatives of trans collectives and the increase of children and adolescents who attend primary care due to gender identity problems. This growing interest has stimulated research whose abundance requires an analysis and selection of the most relevant works. With this objective we have carried out a bibliographic review in order to address the current state of early transsexuality, focusing on studies related to its etiology, prevalence, diagnosis and current treatments. For this, several databases were consulted (Web of Science, PubMed, PubPsych, Elsevier, PsycINFO, PsycARTICLES, Psychology Database) restricting the search by keywords ("transsexuality", "transsexualism", "adolescence", "adolescents" and "teenagers ") and publication date (2008-2017). Initially, 103 were obtained, of which 66 were discarded for treating aspects that were not subject of the review and exposing non-extrapolated results due to their small sample. From the analysis of the 37 selected articles, it is obtained, as regards the etiology, that transsexuality has a heterogeneous and multifactorial origin. The prevalence obtained is $9.8 \%$. The diagnosis insists on the need to carry out a permanent follow-up using the interview as the main instrument of choice. Finally, most research highlights the importance of the new therapeutic approach based on triadic therapy that includes psychological treatment (assimilation of the desired role fully), hormonal (hormonal blocking and subsequent treatment with crossed hormones) and surgical (change of genitals and other sexual characters), in spite of the controversy that has provoked by the possible unfavorable effects for the infantile and juvenile population. It concludes the need to continue research to achieve not only a more individualized diagnosis and treatment, but also a greater knowledge dissemination that helps reduce stigmatization.

Keywords: transexuality; adolescence; transexualizing process

\section{ANTECEDENTES}

La adolescencia es una etapa en la que confluyen aspectos psicobiológicos y socioculturales que conforman la identidad de la persona adolescente y determinan la forma en la que vive su sexualidad y sus vínculos con la salud (Beltrán, Bonilla y Bataller, 2015). Un periodo en el que convergen muchas dificultades, exacerbadas en el caso de los adolescentes transexuales.

La transexualidad no es un fenómeno actual, existe desde muy antiguo en múltiples culturas. Este término fue utilizado por primera vez en 1940 para hacer referencia a los individuos que deseaban vivir de forma permanente como miembros del sexo opuesto, existiendo una incongruencia entre el sexo con el que nacieron y el sexo al que sentían pertenecer. Pero en 1973, aparece un nuevo término, "disforia de género", que incluye el transexualismo y otros trastornos de identidad de género, y designa la insatisfacción que resulta del conflicto entre la identidad de género y el sexo asignado (Sánchez, Mora \& Oviedo, 2017).

Poco más tarde, en 1980, se incluye al "transexualismo" dentro del DSM-III. Un término que con el fin de lograr la despatologización del fenómeno es abandonado y sustituido por "trastorno de 
identidad de género" en el DSM-IV y por "disforia de identidad de género" en el DSM-5 (Sánchez, Mora \& Oviedo, 2017).

Los múltiples cambios que ha sufrido el término en las dos últimas décadas son un reflejo de la importancia de la temática trans que ha emergido como un asunto de interés creciente tanto a nivel social como cultural. Pero detrás de todo este proceso cambiante encontramos, ante todo, las iniciativas de colectivos trans que han ido logrando que las administraciones competentes comiencen a interesarse por sus necesidades y demandas en materia de educación, sanidad y ocupación (Miguel \& García, 2017). Ejemplo de ello, son las leyes de no discriminación por motivos de identidad de género y de reconocimiento a los derechos de las personas transexuales aprobadas recientemente, así como las propuestas de ley de atención sanitaria a personas con disforia de género.

Sumado a todo esto, también se ha detectado un aumento de casos, triplicándose los menores que solicitan atención (Asenjo-Araque et al., 2015). No obstante, se desconoce hasta qué punto este aumento de las derivaciones de menores a servicios especializados responde a un incremento real en la prevalencia, o simplemente refleja una mayor disposición de los jóvenes a "salir del armario" influenciados por los medios sociales (blogs y sitios web) y movimientos asociativos que les ayudan en sus luchas (Wood, et al., 2013).

\section{OBJETIVOS}

El presente trabajo tiene como propósito conocer, desde una perspectiva global, el estado actual de la transexualidad temprana para lo cual se plantea una revisión bibliográfica de los artículos de divulgación científica e investigaciones más recientes que permita explorar y realizar una descripción de su etiología, prevalencia, y abordaje diagnóstico y terapéutico actual.

\section{METODOLOGíA}

Se realizó una búsqueda sistemática en las bases de datos de Web of Science, PubMed, PubPsych, Elsevier, PsycINFO, PsycARTICLES, Psychology Database, de los trabajos publicados en los últimos 10 años (2008-2017) en español e inglés, con los siguientes descriptores: "transsexuality", "transsexualism", "adolescence", "adolescents" y "teenagers", combinados para dar lugar a las siguientes ecuaciones de búsqueda: ("transsexuality" OR "transsexualism”) AND ("adolescence" OR "adolescents" OR "teenagers").

A partir del número inicial de trabajos obtenidos como resultado de las búsquedas en las bases de datos citadas anteriormente $(n=103)$ se hizo un primer cribado para descartar aquellos artículos que fueran estudios de validación de instrumentos o estudios de caso único, quedando la muestra en 64 estudios. Seguidamente, se aplicaron los siguientes criterios de selección: 1) Estar centrados en uno o más aspectos objeto de análisis en nuestra revisión: etiología, epidemiología, diagnóstico, y tratamiento de la transexualidad adolescente; 2) Presentar resultados basados en una muestra lo suficientemente amplia como para extrapolar lo expuesto a toda la población infanto-juvenil. Solo 37 artículos cumplieron todos los criterios y pasaron a conformar la muestra de estudio definitiva.

\section{MUESTRA}

Partiendo inicialmente de 103 artículos, tras la aplicación de los criterios ya indicados en el apartado anterior, resultó una muestra definitiva de 37 artículos.

\section{RESULTADOS ${ }^{1}$}

\section{Etiología}

Del total de artículos seleccionados, 11 exponían teorías sobre el origen de la transexualidad, 


\section{TRANSEXUALIDAD Y ADOLESCENCIA: UNA REVISIÓN SISTEMÁTICA}

coincidiendo en la naturaleza heterogénea y multifactorial de la misma y afirmando que la diferenciación sexual del cerebro humano y el desarrollo de la identidad de género son resultado de una interacción de factores biológicos (genéticos y hormonales) y ambientales (psicosociales). A pesar de ello, las investigaciones analizadas apoyan bien teorías biopsicosociales bien teorías neurobiológicas, de acuerdo con los resultados obtenidos en las mismas.

Por un lado, el $63.6 \%$ de las investigaciones analizadas apuntan hacia explicaciones neurobiológicas. Específicamente, el 27.3\% se presentan como estudios histológicos, basados en la hipótesis de que los cerebros de los adolescentes con disforia de género no siguen la misma línea de diferenciación sexual, siendo programados de forma atípica durante el desarrollo prenatal. Estas investigaciones han recibido el apoyo de los estudios post mortem de cerebros de personas transexuales, que señalan que su núcleo basal de la estría terminal situado en el hipotálamo muestra características similares a los cerebros de personas del sexo biológico opuesto. En cambio, el 36.3\% de los artículos analizados se centraban en el estudio de los genes implicados en la síntesis de esteroides sexuales.

Por otro lado, son pocas las investigaciones que proponen teorías basadas en factores ambientales $(36.4 \%)$. A pesar de ello, el $18.2 \%$ de los estudios extraídos tienen en cuenta el apego para confirmar la asociación entre la vivencia de experiencias traumáticas tempranas y el desarrollo de disforia de género, encontrando entre este colectivo un mayor porcentaje de apego desorganizado 0 inseguro. Respecto al estudio de los rasgos psicológicos y de personalidad, hay estudios (18.2\%) que informan de una alta proporción de comorbilidad psiquiátrica en los sujetos diagnosticados, quedando señalados como posibles causas del transexualismo.

Tabla 1. Investigaciones publicadas entre 2008 y 2017 sobre etiología de la transexualidad.

\begin{tabular}{ll}
\hline \multicolumn{1}{c}{$\begin{array}{c}\text { Factores biológicos } \\
\text { (genéticos y hormonales) }\end{array}$} & \multicolumn{1}{c}{$\begin{array}{c}\text { Factores ambientales } \\
\text { (psicosociales) }\end{array}$} \\
\cline { 2 - 3 } Moreno-Pérez \& De Antonio, 2012 & $\begin{array}{l}\text { Colizzi, Costa, Pace \& Todarello, } \\
2013\end{array}$ \\
Turban \& Ehrensaft, 2017 & Vitelli \& Riccardi, 2011 \\
Berenbaum \& Beltz, 2011 & $\begin{array}{l}\text { de Vries, Steensma, Cohen- } \\
\text { Kettenis, VanderLaan \& Zucker, } \\
\text { Klink \& Den Heijer, 2014 }\end{array}$ \\
Fernández, Esteva et al., 2014 & $\begin{array}{l}\text { Lingiardi, Giovanardi, Fortunato, } \\
\text { Nassisi \& Speranza, 2017 }\end{array}$ \\
Heylens et al., 2012 &
\end{tabular}

$$
\text { \% TOTAL }
$$$$
63.6 \%
$$

$36.4 \%$

\section{Epidemiología}

Realizar estimaciones fiables de la prevalencia del transexualismo temprano resulta muy complicado debido a la falta de estudios epidemiológicos formales y al estigma social imperante en la cultura. En la muestra analizada los estudios epidemiológicos conforman el 18.9\% de los artículos seleccionados, los cuales presentan aproximaciones que varían ampliamente de acuerdo a la metodología usada. A pesar de ello, las investigaciones analizadas muestran prevalencias que oscilan 
entre el $0,003 \%$ y el $5 \%$, proponiendo la mayoría (4) estimaciones por debajo del $1 \%$. A pesar de ello, 5 de los estudios diferenciaban entre el porcentaje de casos de niños transexuales y el de niñas transexuales, aunque no solían darse diferencias significativas $(<1 \%)$.

Sin embargo, cabe resaltar que los 7 estudios recogen el incremento sustancial de casos de transexualidad infantil y adolescente en las dos últimas décadas, así como un aumento progresivo de la prevalencia a medida que aumenta la edad. Al mismo tiempo, aunque las estimaciones varían, coinciden en una mayor incidencia de transexuales femeninos frente a masculinos.

Tabla 2. Investigaciones publicadas entre 2008 y 2017 sobre la epidemiología del transexualismo infanto-juvenil.

\begin{tabular}{llll}
\hline \multicolumn{1}{c}{$<\mathbf{1 \%}$} & Entre 1\%-3\% & \multicolumn{1}{c}{$\mathbf{3 \%}$} \\
\cline { 2 - 4 } & Europapress, 2017 & Shields et al., 2013 & Meyer, 2012 \\
& $\begin{array}{l}\text { Steensma, van der Ende, } \\
\text { Verhulst \& Cohen-Kettenis, }\end{array}$ & Clark et al., 2014 & \\
2012 & & & \\
& WPATH, 2011 & & \\
& Zucker \& Lawrence, 2009 & & \\
\hline \%TOTAL & $\mathbf{5 7 . 1 \%}$ & $\mathbf{2 8 . 6 \%}$ & $\mathbf{1 4 . 3 \%}$ \\
\hline
\end{tabular}

\section{Diagnóstico}

El $35.1 \%$ de los artículos seleccionados disertan sobre el diagnóstico de transexualidad en la adolescencia, coincidiendo en la necesidad de realizar evaluaciones cuidadosas e individualizadas con seguimiento longitudinal en población infanto-juvenil, mediante un abordaje multidisciplinar. A pesar de ello, dadas las consecuencias parcial o totalmente irreversibles de los tratamientos hormonales y quirúrgicos, y la persistencia variable e incierta de este diagnóstico en adolescentes, no todos los autores se muestran favorables a establecer diagnósticos definitivos a edades tempranas. Este grupo de estudios representaría el 38.5\% de los estudios sobre el diagnóstico, apoyando que se trata de un sistema dinámico influenciable por factores disposicionales y socioculturales. Sin embargo, un porcentaje menor de investigaciones (23.1\%) sí confía en los diagnósticos a edades tempranas ya que han encontrado que raramente el diagnóstico se ve modificado entre los menores puberales, siendo los beneficios mucho mayores que las posibles consecuencias.

Por otro lado, los procedimientos de evaluación con niños/as y adolescentes distan mucho de los establecidos para la población adulta. Así, respecto a los procedimientos a usar para realizar un diagnóstico fiable, el $15.4 \%$ de los estudios analizados aluden a la entrevista clínica (estructurada 0 no estructurada) como único instrumento de elección, argumentando que permite realizar una anamnesis pormenorizada que abarca todas las áreas del paciente y su historia psico-biográfica. A pesar de ello, un porcentaje mayor de investigaciones (30.8\%) propone complementar la entrevista clínica con otras técnicas como diarios de actividades cotidianas, registros de ítems descriptivos del estado de ánimo del menor, o valoraciones del malestar que experimenta en su día a día. Estos exponen su utilidad para indagar sobre su actitud frente a la sexualidad (orientación sexual, compulsividad sexual, experiencias sexuales y significado subjetivo de la sexualidad) y su funcionamiento emocional y cognitivo (personalidad, autoestima y autoconcepto). 


\section{TRANSEXUALIDAD Y ADOLESCENCIA: UNA REVISIÓN SISTEMÁTICA}

Tabla 3. Investigaciones publicadas entre 2008 y 2017 sobre el diagnóstico de transexualismo a edad temprana.

\begin{tabular}{|c|c|c|}
\hline & $\begin{array}{l}\text { A favor de un diagnóstico } \\
\text { durante la pubertad }\end{array}$ & $\begin{array}{c}\text { En contra de un diagnostico } \\
\text { durante la pubertad }\end{array}$ \\
\hline & Hembree et al., 2009 & $\begin{array}{l}\text { Fernández, Guerra, Díaz \& } \\
\text { GIDSEEN, } 2014\end{array}$ \\
\hline & Spack et al., 2012 & de Vries \& Cohen-Kettenis, 2013 \\
\hline & \multirow[t]{3}{*}{ Sánchez, Mora \& Oviedo, 2017} & Drescher \& Byne, 2012 \\
\hline & & Wallien \& Cohen-Kettenis, 2008 \\
\hline & & $\begin{array}{l}\text { Drummond, Bradley, Peterson- } \\
\text { Badali, \& Zucker, } 2008\end{array}$ \\
\hline \% TOTAL & $37.5 \%$ & $62.5 \%$ \\
\hline
\end{tabular}

Tabla 4. Investigaciones publicadas entre 2008 y 2017

sobre los instrumentos usados el diagnóstico de transexualismo temprano.

\begin{tabular}{llll}
\hline \multicolumn{2}{l}{ Entrevista clínica (únicamente) } & $\begin{array}{c}\text { Entrevista clínica y otras técnicas } \\
\text { complementarias }\end{array}$ \\
\cline { 2 - 3 } & Asenjo-Araque et al., 2015 \\
2012 & Cohen-Kettenis \& Klink, 2015 & \\
Rodríguez-Molina et al., 2015 & $\begin{array}{l}\text { Fernández, Guerra, Díaz \& } \\
\text { GIDSEEN, 2014 }\end{array}$ \\
\hline \% TOTAL & $\mathbf{3 3 . 3 \%}$ & Sánchez, Mora \& Oviedo, 2017 \\
\hline
\end{tabular}

\section{Tratamiento}

Aunque actualmente las guías de práctica clínica (elaboradas por el GIDSEEN y WPATH) recomiendan un abordaje terapéutico basado en la terapia triádica (intervención psicológica, hormonal y quirúrgica), no todos los estudios exponen una intervención que recoja todos los ámbitos. Así, de los 14 artículos sobre tratamiento analizados, el 39.3\% de ellos hacen hincapié en la atención psicológica, mientras el $60.7 \%$ se centran en la terapia médico-quirúrgica como medio de intervención. 
Tabla 5. Investigaciones publicadas entre 2008 y 2017 sobre el tratamiento en la transexualidad temprana.

\begin{tabular}{lll}
\hline \multicolumn{1}{c}{ Intervención psicológica } & Intervención médico-quirúrgica \\
\cline { 2 - 3 } Basterra-Gortari, 2016 & Olson, Forbes \& Belzer, 2011 \\
Asenjo-Araque et al., 2015 & Fuss, Auer \& Briken, 2015 \\
$\begin{array}{l}\text { Dierckx, Motmans, Mortelmans \& } \\
\text { T'sjoen, 2016 }\end{array}$ & Moreno-Pérez \& De Antonio, 2012 \\
Forcier \& Olson, 2014 & Basterra-Gortari, 2016 \\
Menvielle \& Gómez-Lobo, 2011 & Hembree et al., 2009 \\
Wallien \& Cohen-Kettenis, 2008 & WPATH, 2011 \\
& & Zucker et al., 2011 \\
& & Cohen-Kettenis \& Klink, 2015 \\
\hline \% TOTAL & $\mathbf{3 9 . 3 \%}$ & $\mathbf{6 0 . 7 \%}$ \\
\hline
\end{tabular}

\section{Intervención psicológica}

El proceso transexualizador suele generar malestar psicológico, ocasionando en muchos casos, alteraciones en diversas áreas de funcionamiento (social, familiar, educativo, personal), tal y como exponen 5 de los artículos analizados. Esto justificaría la incorporación del abordaje psicológico como fase esencial del proceso terapéutico tanto para los/as adolescentes que se someten a tratamiento hormonal como aquellos que no lo hacen. A pesar de ello, y debido a la escasez de artículos que tratan el tratamiento psicológico de los adolescentes transexuales (39.3\%), no se han encontrado suficientes estudios que permitan establecer un procedimiento de intervención psicológica estandarizado, puesto que hacen hincapié en diferentes aspectos.

En primer lugar, el $15.4 \%$ de los artículos analizados en este apartado concibe la psicoterapia como una intervención basada en el acompañamiento y apoyo vital al paciente que está experimentando una transición hacia una vivencia normalizada de su identidad sexual. Por ello, la describen como un espacio libre para expresar sentimientos, pensamientos, experiencias, recuerdos y emociones relativas a la auténtica identidad del paciente, ayudándole a alcanzar el máximo confort personal y auto-plenitud, y maximizar el estado de salud y el bienestar psicológico.

En segundo lugar, el $7.7 \%$ de los estudios considera la psicoterapia como un espacio para el asesoramiento del menor de edad con problemas en su identidad de género, aportándole la información suficiente sobre el tratamiento hormonal y farmacológico como para permitirle conocer y valorar los beneficios y riesgos de cada uno de ellos.

Por otro lado, representado por un $15.4 \%$ se han encontrado estudios que plantean como función principal del tratamiento psicológico la intervención en el entorno social y familiar del menor, debido a su relevante papel en el desarrollo infanto-juvenil. Por ello, resaltan la coordinación con el centro académico para asegurar la integración del menor y manejar situaciones de transfobia, así como el abordaje con la familia para tratar el impacto de tener un hijo/a transexual y dar soporte e 


\section{TRANSEXUALIDAD Y ADOLESCENCIA: UNA REVISIÓN SISTEMÁTICA}

información ayudando a mejorar su comprensión de la situación del menor consiguiendo su implicación en el proceso.

Tabla 6. Investigaciones publicadas entre 2008 y 2017

sobre la intervención psicológica en la transexualidad temprana.

\begin{tabular}{lll}
\hline $\begin{array}{c}\text { Intervención psicológica } \\
\text { centrada en el paciente }\end{array}$ & $\begin{array}{c}\text { Intervención psicológica centrada } \\
\text { en el entorno sociofamiliar }\end{array}$ \\
\cline { 2 - 3 } Basterra-Gortari, 2016 & Dierckx et al., 2016 \\
Asenjo-Araque et al., 2015 & Forcier \& Olson, 2014 \\
Menvielle \& Gómez-Lobo, 2011 & \\
& Wallien \& Cohen-Kettenis, 2008 & \\
\hline \% TOTAL & $\mathbf{6 0 \%}$ & $\mathbf{4 0 \%}$ \\
\hline
\end{tabular}

\section{Intervenciones médicas}

El deseo de vivir y ser aceptado como miembro del género opuesto puede estar acompañado en algunos jóvenes del deseo de modificar el propio cuerpo para hacerlo lo más congruente posible con su identidad de género. Con ello, el $60.7 \%$ de los artículos que analizan el tratamiento de los adolescentes transexuales proponen un tratamiento basado en las intervenciones médico-quirúrgicas.

Entre ellos, la mayoría (37.5\%) plantean como intervención de elección el tratamiento mediante la supresión del eje gonadal en los estadíos iniciales del desarrollo puberal. Una técnica reversible que consiste en la administración de análogos de GnRH que bloquean la pubertad hormonal en su sexo biológico.

Pero existen opiniones controvertidas respecto a este tratamiento, ya que no todos los autores consideran a esta como una técnica recomendable. Así, el $66.7 \%$ de los estudios postulan que los beneficios de este tratamiento están bien establecidos y el uso del GnRH se considera seguro y eficaz. Con ello, defienden que este tratamiento permite prevenir el daño psicológico asociado al desarrollo de unos caracteres sexuales secundarios indeseados y proporcionar al adolescente la tranquilidad y el tiempo necesario para explorar su identidad de género y el deseo de realizar un cambio corporal irreversible, consiguiendo una adaptación psicosocial más adecuada y mejores resultados físicos. Por el contrario, una minoría de estudios (5.5\%) exponen que este tratamiento no debería de ser aplicado hasta que cuente con una mayor validez clínica, ya que puede desencadenar la detención de la mineralización ósea, la altura, el metabolismo y la desproporción de la segmentación del cuerpo, así como la perturbación del desarrollo cerebral (especialmente corteza prefrontal).

Por otro lado, el $25 \%$ de los estudios exponen otras alternativas como son el tratamiento con hormonas sexuales cruzadas (a partir de los 16 años) y la cirugía de reasignación de sexo (al llegar a los 18 años). Bien cabe matizar que son recomendados en aquellos casos en que el malestar sobre la imagen corporal persiste sin producirse variaciones en cuanto a la identidad de género. 
Tabla 7. Investigaciones publicadas entre 2008 y 2017

sobre la intervención médico-quirúrgica en la transexualidad temprana.

\begin{tabular}{|c|c|c|c|}
\hline & Tratamiento mediante a & nálogos de GnRH & \\
\hline & Simpatizantes & Detractores & $\begin{array}{c}\text { miervenciones meaco- } \\
\text { quirúrgicos }\end{array}$ \\
\hline & WPATH, 2011 & $\begin{array}{l}\text { Moreno-Pérez \& } \\
\text { De Antonio, } 2012\end{array}$ & $\begin{array}{l}\text { Moreno-Pérez \& De } \\
\text { Antonio, } 2012\end{array}$ \\
\hline & Zucker et al., 2011 & & $\begin{array}{l}\text { Fuss, Auer \& Briken, } \\
2015\end{array}$ \\
\hline & Hembree et al., 2009 & & Basterra-Gortari, 2016 \\
\hline & $\begin{array}{l}\text { Cohen- } \\
\text { Kettenis \& Klink, } 2015\end{array}$ & & \\
\hline & Esteva et al., 2015 & & \\
\hline & $\begin{array}{l}\text { Olson, Forbes \& } \\
\text { Belzer, } 2011\end{array}$ & & \\
\hline & $66.7 \%$ & $5.5 \%$ & \\
\hline$\%$ TOTAL & $72.2 \%$ & & $27.8 \%$ \\
\hline
\end{tabular}

\section{DISCUSIÓN Y CONCLUSIONES}

A pesar de la escasez de información sobre el transexualismo en población infanto-juvenil, los 37 artículos seleccionados evidencian que el incremento del número de casos de adolescentes transexuales que llegan a las Unidades de Identidad de Género ha supuesto un pronunciado movimiento científico con impacto a diferentes niveles.

Así, el $37.8 \%$ de los artículos escogidos analizan la atención terapéutica proporcionada a este sector de la población, confirmando el incremento de los servicios ofertados mediante la inclusión del bloqueo hormonal. Esto ha supuesto un aumento de la esperanza de conseguir una mejora sustancial en el bienestar y calidad de vida de los adolescentes transexuales. Lo mismo sucede con la creación de guías prácticas con recomendaciones sobre los estándares de cuidados de las personas transexuales, que introducen la necesidad de tener en cuenta el abordaje triádico: psicológico, hormonal y quirúrgico.

Al mismo tiempo, este incremento de la demanda ha puesto en relieve la importancia de realizar un abordaje específico y especializado teniendo en cuenta las dificultades de los niños/as y adolescentes para expresar su estado y su desarrollo cambiante, inestable e inmaduro. Esto provoca que el diagnóstico se convierta en un procedimiento necesariamente documentado, según exponen el $35.1 \%$ de los estudios revisados.

Aun así, son varios aspectos los que demandan mayor dedicación científica, ya que, aunque el $29.7 \%$ de los artículos que conforman nuestro estudio indagan sobre el origen del transexualismo, especialmente sobre las teorías neurobiológicas, siguen siendo escasas las investigaciones que extraen conclusiones esclarecedoras. Algo parecido sucede con los estudios sobre la prevalencia del transexualismo en este colectivo (representando el 18.9\% de nuestra muestra), debido en gran parte 


\section{TRANSEXUALIDAD Y ADOLESCENCIA: UNA REVISIÓN SISTEMÁTICA}

a lo novedoso de la atención profesionalizada en salud pública que justifica la escasez de datos y de estudios longitudinales.

Pero realizar análisis casuísticos compartiendo conocimientos empíricos no solo es necesario por su contribución a nivel clínico, sino también a nivel social. Por un lado, la controversia entre los defensores de las intervenciones médicas y sus detractores sigue latente, pudiéndose esclarecer mediante estudios de seguimiento que desvelen si realmente tiene más consecuencias negativas que positivas. Por otro lado, el desconocimiento social nos lleva al miedo y al prejuicio, por lo que la divulgación de conocimiento podría contribuir a la normalización de las vivencias transexuales promoviendo la simpatía hacia la diversidad social.

\section{REFERENCIAS BIBLIOGRÁFICAS}

Asenjo-Araque, N., García-Gibert, C., Rodríguez-Molina, J.M., Becerra-Fernández, A., Lucio-Pérez, M.J., \& GIDSEEN, G. (2015). Disforia de género en la infancia y adolescencia: una revisión de su abordaje, diagnóstico y persistencia. Revista de Psicología Clínica con Niños y Adolescentes, 2(1), 33-36.

Basterra-Gortari, V. (2016). Unidad Navarra de Transexuales e Intersexos: atención a la disforia de género en población infantil y adolescente. Boletín de la Sociedad Vasco-Navarra de pediatría, (117), 20-22.

Beltrán, A., Bonilla, A. y Bataller, V. (2015) Sexualidad y salud sexual en la construcción de las identidades de género y la orientación del deseo sexual en adolescentes (Tesis doctoral). Universitat de Valencia, Valencia.

Bentz, E.K., Hefler, L.A., Kaufmann, U., Huber, J.C., Kolbus, A., \& Tempfer, C.B. (2008). A polymorphism of the CYP17 gene related to sex steroid metabolism is associated with female-to-male but not male-to-female transsexualism. Fertility and sterility, 90(1), 56-59.

Berenbaum, S.A., \& Beltz, A.M. (2011). Sexual differentiation of human behavior: effects of prenatal and pubertal organizational hormones. Frontiers in neuroendocrinology, 32(2), 183-200.

Clark, T.C., Lucassen, M.F., Bullen, P., Denny, S.J., Fleming, T.M., Robinson, E.M., \& Rossen, F.V. (2014). The health and well-being of transgender high school students: Results from the New Zealand adolescent health survey (Youth'12). Journal of Adolescent Health, 55, 93-99.

Cohen-Kettenis, P.T. \& Klink, D. (2015) Adolescents with gender dysphoria. Best Practice \& Research Clinical Endocrinology \& Metabolism, 29, 485-495.

Colizzi, M., Costa, R., Pace, V., \& Todarello, 0. (2013). Hormonal treatment reduces psychobiological distress in gender identity disorder, independently of the attachment style. Journal of Sexual Medicine, 10, 3049-3058.

de Vries, A.L., \& Cohen-Kettenis, P.T. (2013). Clinical management of gender dysphoria in children and adolescents: the Dutch approach. Journal of homosexuality, 59(3), 301-320.

de Vries, A.L., Steensma, T.D., Cohen-Kettenis, P.T., VanderLaan, D.P.,\&Zucker, K.J. (2016). Poor peer relations predict parent- and self-reported behavioral and emotional problems of adolescents with gender dysphoria: A cross-national, cross-clinic comparative analysis. European Child and Adolescent Psychiatry, 25, 579-588.

Dierckx, M., Motmans, J., Mortelmans, D., T'sjoen, G. (2016) Families in transition: A literature review. International Review Psychiatry, 28(1): 36-43.

Drummond, K.D., Bradley, S.J., Peterson-Badali, M., \& Zucker, K.J. (2008). A follow-up study of girls with gender identity disorder. Developmental psychology, 44(1), 34.

Drescher, J. \& Byne, W. (2012). Gender dysphoric/gender variant (GD/GV) children and adolescents: Summarizing what we know andwhat we have yet to learn. Journal of Homosexuality, 59, 501-510. 
Esteva, I., Asenjo, N., Hurtado, F., Fernández, M., Vidal, A., Moreno-Pérez, Ó., Lucio, M.J., López, J.P. \& GIDSEEN (2015) Documento de posicionamiento: disforia de género en la infancia y la adolescencia. Grupo de Identidad y Diferenciación Sexual de la Sociedad Española de Endocrinología y Nutrición (GIDSEEN). Endocrinol Nutr. 62(8), 380-383.

EuropaPress (1 enero 2017). La Unidad de Transexualidad del Peset atiende a 24 menores en 2016 y realizará en enero la primera vaginoplastia. La Vanguardia. Recuperado de http://www.lavanguardia.com

Fernández, M., Guerra, P., Díaz, M. \& GIDSEEN (2014). La disforia de género en la infancia en las clasificaciones diagnósticas. Cuadernos de medicina psicosomática y psiquiatría de enlace, (110), 25-35.

Fernández, R., Esteva, I., Gómez Gil, E., Rumbo, T., Almaraz, M.C., Roda, E., Haro-Mora, J.J., Guillamón, A. \& Pásaro, E. (2014). The (CA) n polymorphism of ER gene is associated with FtM transsexualism. The journal of sexual medicine, 11(3), 720-728.

Forcier M, \& Olson J. (2014) Overview of gender development and clinical presentation of gender nonconformity in children and adolescents. UpToDate.

Fuss, J., Auer, M.K., \& Briken, P. (2015). Gender dysphoria in children and adolescents: a review of recent research. Curr Opin Psychiatry, 28(6), 430-434.

Hembree, W.C., Cohen-Kettenis, P., Delemarre-Van De Waal, H.A., Gooren, L.J., Meyer III, W.J., Spack, N.P., Tangpricha, V. \& Montori, V.M. (2009). Endocrine treatment of transsexual persons: an Endocrine Society clinical practice guideline. The Journal of Clinical Endocrinology \& Metabolism, 94(9), 3132-3154.

Heylens, G., De Cuypere, G., Zucker, K.J., Schelfaut, C., Elaut, E., Vanden Bossche, H., De Baere, E. \& T'sjoen, G. (2012). Gender identity disorder in twins: a review of the case report literature. Journal of sexual medicine, 9(3), 751-757.

Klink, D., \& Den Heijer, M. (2014). Genetic aspects of gender identity development and gender dysphoria. In: Kreukels BPC, Steensma TD, Vries de ALC, editors. Gender dysphoria and disorders of sex development. 1st ed. New York, US: Springer; 2013, 25-51.

Lingiardi, V., Giovanardi, G., Fortunato, A., Nassisi, V., \& Speranza, A.M. (2017). Personality and attachment in transsexual adults. Archives of sexual behavior, 46(5), 1313-1323.

Menvielle E, \& Gomez-Lobo V. (2011) Management of children and adolescents with gender dysphoria. J Pediatr Adolesc Gynecol; 24:183.

Meyer, W.J. (2012) Gender identity disorder: an emerging problem for pediatricians. Pediatrics; 129:571.

Miguel, J.F., \& García, S.P. (2017). Transexualidad, adolescencias y educación: miradas multidisciplinares, de Octavio Moreno Cabrera y Luis Puche Cabezas. AIBR, Revista de Antropología Iberoamericana, 12(1), 103-108.

Moreno-Pérez, Ó., De Antonio, I.E., \& GIDSEEN. (2012). Clinical practice guidelines for assessment and treatment of transsexualism. SEEN Identity and Sexual Differentiation Group (GIDSEEN). Endocrinol Nutr, 59(6), 367-382.

Olson, J., Forbes, C., \& Belzer, M. (2011). Management of the transgender adolescent. Archives of pediatrics \& adolescent medicine, 165(2), 171-176.

Rodríguez-Molina, J.M., Asenjo-Aranque, N., Becerra-Fernández, A., Lucio-Pérez, M.J., RabitoAlcón, M.F. \& Pérez-López, G. (2015). Áreas de la entrevista para la evaluación psicológica de personas transexuales. Acción Psicológica, 12(2), 15-30.

Sánchez, I., Mora, J.J. \& Oviedo, O. (2017) Psychomedical care in gender identity dysphoria during adolescence. Rev Psiquiatr Salud Ment, 10, 96-103. 


\section{TRANSEXUALIDAD Y ADOLESCENCIA: UNA REVISIÓN SISTEMÁTICA}

Shields, J.P., Cohen, R., Glassman, J.R., Whitaker, K., Franks,H., \& Bertolini, I. (2013). Estimating population size and demographic characteristics of lesbian, gay, bisexual, and transgender youth in middle school. Journal of Adolescent Health, 52, 248-250.

Spack, N.P., Edwards-Leeper, L., Feldman, H.A., Leibowitz, S., Mandel, F., Diamond, D.A., \& Vance, S.R. (2012). Children and adolescents with gender identity disorder referred to a pediatric medical center. Pediatrics.

Steensma TD, van der Ende J, Verhulst FC, Cohen-Kettenis PT. (2012) Gender Variance in Childhood and Sexual Orientation in Adulthood: A Prospective Study. J Sex Med.

The World Professional Association for Transgender Health. (2011) Standards of Care for the Health of Transsexual,Transgender, and Gender Nonconforming People Version 7. International Journal of Transgenderism; 13: 165-232.

Turban, J.L., \& Ehrensaft, D. (2017). Research Review: Gender identity in youth: treatment paradigms and controversies. Journal of Child Psychology and Psychiatry.

Vitelli, R., \& Riccardi, E. (2011). Gender identity disorder and attachment theory: The influence of the patient's internal working models on psychotherapeutic engagement and objective. A study undertaken using the Adult Attachment Interview. International Journal Transgenderism, 12, 241-253.

Wallien, M.S., Cohen-Kettenis, P.T. (2008) Psychosexual outcome ofgender-dysphoric children. $J$ Am Acad Child Adolesc Psychiatry, 47:1413-23.

Wood, H., Sasaki, S., Bradley, S.J., Singh, D., Fantus, S., Owen-Anderson, A., Zucker, K.J. (2013). Patterns of referral to a gender identity service for children and adolescents (1976-2011): Age, sex ratio, and sexual orientation. Journal of Sex and Marital Therapy, 39, 1-6.

Zucker, K.J., Bradley, S.J., Owen-Anderson, A., Singh, D., Blanchard, R., \& Bain, J. (2011). Pubertyblocking hormonal therapy for adolescents with gender identity disorder: A descriptive clinical study. Journal of Gay \& Lesbian Mental Health, 15(1), 58-82.

Zucker, K.J. \& Lawrence, A.A. (2009). Epidemiology of gender identity disorder: Recommendations for the standards of care of The World Professional Association for Transgender Health. International Journal Transgenderism, 11(1),8-18. 


\section{ANEXO 1.}

Tabla 8. Relación de artículos vinculados a cada uno de los apartados expuestos en Resultados.

\begin{tabular}{|c|c|c|c|c|}
\hline & Etiol. & Epid. & Diagn. & Trat. \\
\hline Asenjo-Araque et al., 2015 & & & $\mathrm{X}$ & $\mathrm{X}$ \\
\hline Basterra-Gortari, 2016 & & & & $\mathrm{X}$ \\
\hline Bentz et al., 2008 & $\mathrm{X}$ & & & \\
\hline Berenbaum \& Beltz, 2011 & $\mathrm{X}$ & & & \\
\hline Clark et al., 2014 & & $\mathrm{X}$ & & \\
\hline Cohen-Kettenis \& Klink, 2015 & & & $X$ & $X$ \\
\hline Colizzi, Costa, Pace \& Todarello, 2013 & $\mathrm{X}$ & & & \\
\hline de Vries \& Cohen-Kettenis, 2013 & & & $\mathrm{X}$ & \\
\hline de Vries, Steensma, Cohen-Kettenis, VanderLaan \& Zucker, 2016 & $\mathrm{X}$ & & & \\
\hline Dierckx et al., 2016 & & & & $X$ \\
\hline Drescher \& Byne, 2012 & & & $\mathrm{X}$ & \\
\hline Drummond, Bradley, Peterson-Badali \& Zucher, 2008 & & & $X$ & \\
\hline Esteva et al., 2015 & & & & $\mathrm{X}$ \\
\hline Europapress, 2017 & & $X$ & & \\
\hline Fernández, Guerra, Díaz \& GIDSEEN, 2014 & & & $\mathrm{X}$ & \\
\hline Fernández, Esteva et al., 2014 & $\mathrm{X}$ & & & \\
\hline Forcier \& Olson, 2014 & & & & $X$ \\
\hline Fuss, Auer \& Briken, 2015 & & & & $\mathrm{X}$ \\
\hline Hembree et al., 2009 & & & $\mathrm{X}$ & $\mathrm{X}$ \\
\hline Heylens et al., 2012 & $X$ & & & \\
\hline Klink \& Den Heijer, 2014 & $\mathrm{X}$ & & & \\
\hline Lingiardi, Giovanardi, Fortunato, Nassisi \& Speranza, 2017 & $\mathrm{X}$ & & & \\
\hline Menvielle \& Gómez-Lobo, 2011 & & & & $\mathrm{X}$ \\
\hline Meyer, 2012 & & $\mathrm{X}$ & & \\
\hline Moreno-Pérez \& De Antonio, 2012 & $\mathrm{X}$ & & $\mathrm{X}$ & $\mathrm{X}$ \\
\hline Olson, Forbes \& Belzer, 2011 & & & & $\mathrm{X}$ \\
\hline Rodríguez-Molina et al., 2015 & & & $\mathrm{X}$ & \\
\hline Sánchez, Mora \& Oviedo, 2017 & & & $\mathrm{X}$ & \\
\hline Shields et al., 2013 & & $\mathrm{X}$ & & \\
\hline Spack et al., 2012 & & & $\mathrm{X}$ & \\
\hline Steensma, van der Ende, Verhulst \& Cohen-Kettenis, 2012 & & $\mathrm{X}$ & & \\
\hline Turban \& Ehrensaft, 2017 & $X$ & & & \\
\hline Vitelli \& Riccardi, 2011 & $\mathrm{X}$ & & & \\
\hline Wallien \& Cohen-Kettenis, 2008 & & & $\mathrm{X}$ & $X$ \\
\hline WPATH, 2011 & & $\mathrm{X}$ & & $\mathrm{X}$ \\
\hline Zucker et al., 2011 & & & $\mathrm{X}$ & $\mathrm{X}$ \\
\hline Zucker \& Lawrence, 2009 & & $\mathrm{X}$ & & \\
\hline PORCENTAJE & 29.7 & 18.9 & 35.1 & 37.8 \\
\hline
\end{tabular}


